\title{
An analysis of calendar performance in two autistic calendar savants
}

\author{
Daniel P. Kennedy ${ }^{1}$ and Larry R. Squire ${ }^{1,2,3,4}$ \\ ${ }^{1}$ Department of Neurosciences, University of California-San Diego, La Jolla, California 92093, USA; ${ }^{2}$ Veterans Affairs Medical \\ Center, San Diego, California 92161, USA; ${ }^{3}$ Departments of Psychiatry and Psychology, University of California-San Diego, \\ La Jolla, California 92093, USA
}

\begin{abstract}
We acquired large data sets of calendar performance from two autistic calendar savants, DG and RN. An analysis of their errors and reaction times revealed that (1) both individuals had knowledge of calendar information from a limited range of years; (2) there was no evidence for the use of memorized anchor dates that could, by virtue of counting away from the anchors, allow correct responses to questions about other dates; and (3) the two individuals differed in their calendar knowledge, as well as in their ability to perform secondary tasks in which calendar knowledge was assessed indirectly. In view of the fact that there are only 14 possible annual calendars, we suggest that both savants worked by memorizing these 14 possible calendar arrangements.
\end{abstract}

Autism is a neurodevelopmental disorder characterized by impairments in social and communicative abilities, together with the presence of restricted and repetitive interests and behaviors. Approximately $10 \%$ of individuals with autism have striking islands of ability (i.e., savant abilities), usually in the domains of memory, calculation, music, or drawing. The most common type of autistic savant is the calendar savant. Calendar savants can provide the day of the week for dates within some particular range of years (e.g., the day of the week for June 6, 1977). This remarkable ability is most commonly found in autistic individuals but has also been reported for non-autistic individuals, usually with below average cognitive functioning ( $\mathrm{O}^{\prime}$ Connor and Hermelin 1984). Interestingly, although there is wide variation in the calendrical abilities of savants (ranging from knowledge of a few years and reaction times of tens of seconds [Mottron et al. 2006] to knowledge of thousands of years and subsecond reaction times [Thioux et al. 2006]), there is no evidence of a relationship between calendar ability and intelligence ( $\mathrm{O}^{\prime}$ Connor and Hermelin 1984).

Despite several investigations of calendar savants, the methods used by savants to determine dates remain poorly understood. Several algorithms exist for determining the day of the week for any given date. However, the idea that these are widely used has been largely dismissed because the impaired arithmetic ability of the typical calendar savant makes it unlikely that the task could be approached in this way. Furthermore, many savants are able to answer "reverse" calendar questions (e.g., "What is the date of the second Monday of March 1983?"), which would require either a substantial modification to these algorithms or a slower method based on generating possible answers and then working toward the correct date (Mottron et al. 2006). A more likely alternative is that the savant uses either memory alone or a combination of memory and simple calculation to take advantage of regularities in the structure of the calendar.

One example of a regularity in the calendar is that its structure repeats every $28 \mathrm{yr}$ (e.g., the dates of 1998 are identical to the dates of 1970). Note that there are only 14 unique calendar templates (i.e., the year can be a leap year or non-leap year, and January 1 can occur on one of seven days of the week). In fact,

${ }^{4}$ Corresponding author.

E-mail Isquire@ucsd.edu; fax (858) 552-7457.

Article is online at http://www.learnmem.org/cgi/doi/10.1101/lm.653607. several autistic savants have explicitly verbalized the 28 -yr regularity, or have been observed to write down successive additions or subtractions of $28 \mathrm{yr}$ until arriving at the correct answer (O'Connor and Hermelin 1984; Ho et al. 1991). Performance by savants that can cover a large range of years (e.g., hundreds or thousands of years) must be supported by knowledge of these calendar regularities. In a model developed to explain the performance of the calendar savant Donny, Thioux et al. (2006) proposed that his abilities, which extend from year 1 A.D. to 9999 A.D., are based on "a large foundation of memorized dateweekday associations, arithmetic, and some knowledge of the rules of calendars."

The calendar ability of some savants covers only a limited set of years. These individuals may lack knowledge of the 28-yr pattern and depend more exclusively on memory or on a combination of memory and other patterns in the calendar. For example, one might learn several "anchor" dates for each month or year and then calculate from these dates using calendar patterns known to everyone. Even a non-savant could determine that if October 1, 2007 is a Monday, then October 10 must be a Wednesday. There is some evidence for the use of such "anchor" strategies. One savant (TM) "could be heard verbally working things out, appearing to start from anchor dates and explaining rules about calendar regularities as he proceeded" (Young and Nettelbeck 1994). Two other savants exhibited faster reaction times for December dates (Rosen 1981), an observation consistent with the use of anchor dates. Yet, faster reaction time and/or increased accuracy for certain parts of the calendar are not strong evidence for an anchor-based strategy. Better performance on some dates might be explained by differences in what parts of the calendar have been most practiced or by differences in the salience of particular calendar features.

Two different approaches have been primarily used to study the basis of the abilities demonstrated by calendar savants. The first approach has been to examine reaction times (RT) and errors to pre-selected (i.e., nonrandom) dates, chosen to test specific hypotheses about potential strategies used by savants (e.g., Hermelin and O'Connor 1986; Young and Nettelbeck 1994). This approach is complicated by the possibility that the savant might recognize and take advantage of structure and regularities within the task. One would then not be sure that the savant uses the same method outside the design of the task. The second approach has been to ask for the day of the week for randomly 
selected dates and then look for patterns in the reaction times (RT) and errors (e.g., Hill 1975; Rosen 1981; O'Connor and Hermelin 1984; Mottron et al. 2006; Thioux et al. 2006; Iavarone et al. 2007). For example, if RTs were always shorter for the first day of the week, and if a systematic increase in RTs were observed as one moved away from that day, one could conclude that the savant is using memorized anchor dates to obtain correct answers for other days of the week. This approach has the advantage that strategies not explicitly tested for might be revealed in the data. Although this approach has been widely used, most studies have acquired a limited number of sparsely sampled observations (e.g., 45 dates [O'Connor and Hermelin 1984]; 192 dates [Rosen 1981]; $\sim 240$ dates [Iavarone et al. 2007]), reducing the possibility of finding patterns in the data (for exceptions, see Mottron et al. 2006; Thioux et al. 2006).

In this study, we sought to identify strategies underlying the calendar abilities of two autistic savants, DG and RN. We acquired large and densely sampled data sets (>1500 dates), and we analyzed the accuracy and reaction time of the responses. We supposed that, if the savants were using an anchor-based strategy, this strategy would be reflected in both faster reaction times and increased accuracy for a particular day of the week, day of the month, or month. Furthermore, we would also expect to find increased error rate and reaction time with increasing distance from these anchor points. In contrast, if a strategy based more exclusively on memory were being used, then we would expect not to find these patterns. We also carried out additional tasks with each savant to explore the flexibility and organization of their calendar knowledge.

\section{Results}

\section{Calendar dates}

\section{DG}

For the period 1993-1998 (tested in 1997-1998), DG scored $81.9 \%$ correct and had a mean reaction time of $4.61 \mathrm{sec}$ (Fig. 1A). Correct answers tended to be given more quickly than incorrect answers (4.56 sec vs. $\left.4.84 \mathrm{sec} ; t_{(1397)}=1.89, P=0.059\right)$. When testing began, DG's calendar knowledge was limited to the period 1993-1998. In fact, DG was unable to provide accurate dates for 1993 during the first seven sessions (1 yr) of testing, but in the eighth session ( $2.5 \mathrm{wk}$ after the seventh session), his performance on dates from 1993 improved from 36.8\% to 95.5\% correct. In addition, when he was tested with an extended list of years 4-6 yr later, DG performed well on a wider range of years (19852007; tested in 2001 and 2003). He now averaged 93.7\% correct across this 22-yr span, although his knowledge was still limited.
Thus, he scored only $27.3 \%$ correct for the years $1979-1984$ and $30.8 \%$ correct for the years 2008-2018. The analyses that follow were based on the years 1993-1998 because these years were sampled most thoroughly.

On average, DG's performance was more accurate for the first day of the month $(96.1 \%$ correct $)$ than for all other days of the month $\left(81.4 \%\right.$ correct; $\left.\chi^{2}[1]=7.17, P<0.01\right)$ (Fig. $\left.2 \mathrm{~A}\right)$. He also responded faster to dates on the first day of the month than to all other dates $\left(3.11 \mathrm{sec} v\right.$ s. $\left.4.67 \mathrm{sec} ; t_{(61)}=8.13, P<0.001\right)$. The last day of the month had the next highest accuracy (91.2\%; $\left.\chi^{2}[1]=3.48, P=0.062\right)$ and the next fastest reaction time compared to all other dates $\left(3.79 \mathrm{sec} ; t_{(65)}=3.98, P<0.001\right)$. (Note that these effects for the last day of the month are not directly apparent in Figure 2A because the figure does not account for whether the last day of the month was on the 28th, 29th, 30th, or 31st day.) It is of interest that the data reveal no gradient in latency or accuracy as one asks about dates that are increasingly distant from the first and last days of the month. For example, there was no linear trend in reaction time or error rate across the first part of the month (days 2-8) or the last part of the month (days 24-30) (all $P$-values $>0.30$ ). DG's performance was similar across the months of the year (accuracy: $F_{(6,1387)}=1.52, P>0.1$; reaction time: $F_{(11,1387)}=0.59, P>0.1$ ) (Fig. $2 \mathrm{~B}$ ). Similarly, his performance was similar across the days of the week (accuracy: $F_{(6,1392)}=1.33, P>0.1$; reaction time: $\left.F_{(6,1392)}=0.83, P>0.1\right)$ (Fig. 2C).

As mentioned, DG learned the calendar year 1993 during the time that we tested him. Interestingly, he learned the days of the week for the first day and last day of each month before he learned the other dates. Thus, in the first seven sessions, when he scored poorly overall for 1993 (26.1\% correct), he scored 83.3\% correct and $50.0 \%$ correct on questions involving the first day and last day of the month, respectively. Furthermore, across these seven sessions, accuracy for dates in months in which the first or last days were answered incorrectly $(24.4 \%$ correct) was not different from accuracy for dates in months in which the first or last days were answered correctly $\left(22.2 \% ; t_{(151)}=0.3 ; P>0.75\right)$.

\section{RN}

For the period 1971-1999 (tested in 1997-1999), RN scored $78.0 \%$ correct and had a mean reaction time of $5.50 \mathrm{sec}$ (Fig. 1B). Like DG, RN gave his correct answers faster than his incorrect answers $\left(5.26 \mathrm{sec}\right.$ vs. $\left.6.34 \mathrm{sec} ; t_{(1728)}=7.8, P<0.001\right)$. RN's knowledge of dates extended beyond the years that we sampled most completely. Thus, he scored $67.9 \%$ correct for the years 1961-1970 and $62.0 \%$ for the years $2000-2007$. Even so, his knowledge was limited. He scored only $26.8 \%$ and $16.5 \%$ correct for the periods 1900-1960 and 2008-2020, respectively. The
A

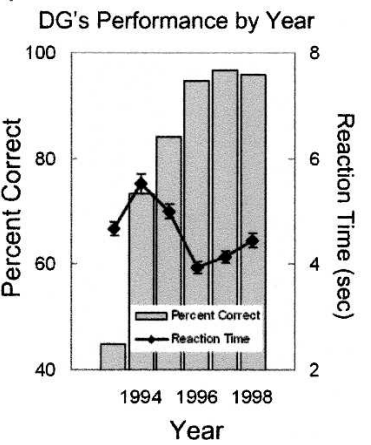

B

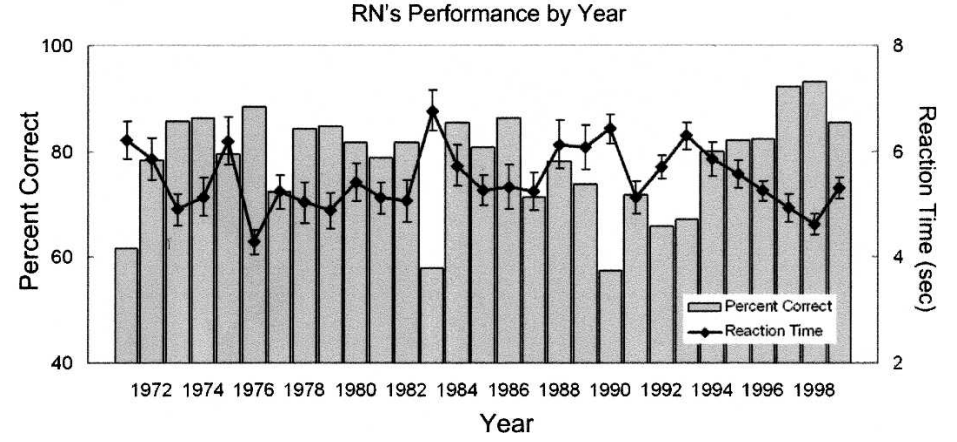

Figure 1. (A) DG's performance by year (reaction time and percent correct). At the time of testing (1997-1998), DG was 9-10 yr old. Each data point shows his mean performance for 197-204 dates. (B) RN's performance by year (reaction time and percent correct). At the time of testing (1997-1999), RN was 33-35 yr old. Each data point shows his mean performance for 38-108 dates. Brackets show standard error of the mean. 
A

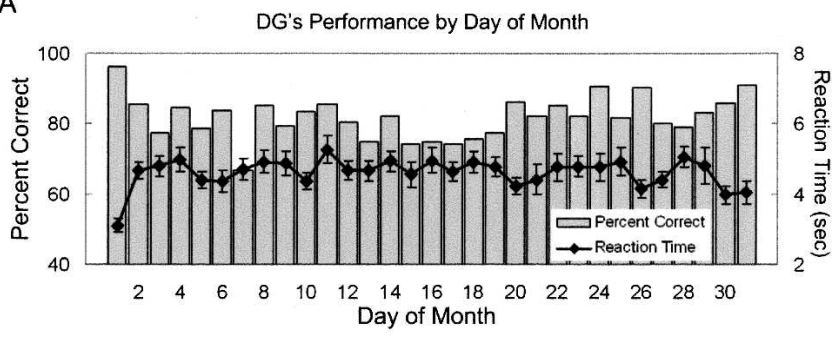

B

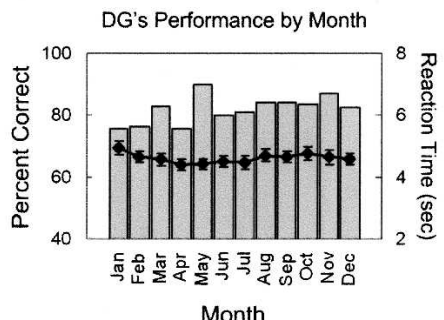

Month
C

DG's Performance by Day of Week

Day of Week

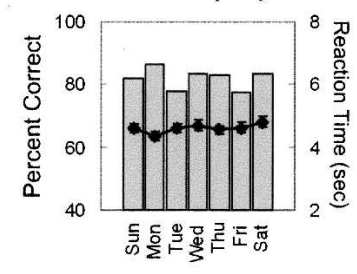

Figure 2. $D G^{\prime}$ 's performance (reaction time and percent correct) by day of the month $(A)$, month of the year $(B)$, and day of the week (C). Note in $A$ the higher accuracy and lower reaction time for the first day of the month compared to the other days of the month. Brackets show standard error of the mean.

analyses that follow were based on the years 1971-1999, because these years were sampled most thoroughly.

RN's performance was similar across the days of the month (Fig. 3A). Unlike DG, he scored about the same on questions involving the first day of the month as on questions involving all other days $\left(\chi^{2}[1]=1.17, P>0.1\right)$ (Fig. $\left.3 A\right)$. Similarly, RN scored about the same on questions about the last day of the month as on questions about the other days $\left(\chi^{2}[1]=0.01, P>0.1\right)$. Figure 3B shows RN's performance by month. Note that he had difficulty with dates in May, June, and July. His score for dates in these three months $(58.1 \%)$ was lower than his score for the other months $(84.6 \%)\left(\chi^{2}[1]=132.14, P<0.001\right)$. Similarly, $\mathrm{RN}^{\prime} \mathrm{s}$ reaction time for dates in these three months $(6.33 \mathrm{sec})$ was significantly slower than his reaction time for dates from the other months [5.22 sec; $\left.t_{(664)}=7.87, P<0.001\right]$. Figure $3 \mathrm{C}$ shows that his accuracy was similar across the days of the week $\left(F_{(6,1723)}=1.10, P>0.1\right)$. There was a small, but significant effect of the day of the week on reaction time $\left(F_{(6,1723)}=2.31, P=0.03\right)$, reflecting the fact that he was a little faster at answering questions involving the beginning or end of the week (i.e., Sunday and Saturday).

\section{Date pairs}

Two dates from the same year and their corresponding days of the week were read aloud to the participants. Following this, participants were asked to answer other date questions from that same year. DG was $98.7 \%$ accurate on this task, answering 78 of 79 questions correctly (chance $=14.3 \%$ ). He took only a few seconds to provide each answer. His mean confidence rating was 5.0. Additionally, DG was given one impossible date pair (i.e., two dates and corresponding days of the week that never appear together in the same year). DG took an unusually long amount of time to respond. As a prompt, he was asked, "Is it possible?" He responded, "Yeah, I'm thinking." After a few seconds, he said, "Wait, it isn't possible." In contrast, RN was unable to perform this task. He answered only $18.3 \%$ of the questions correctly $(11 / 60)$ (chance $=14.3 \%, P>0.1$; binomial test) with a mean

confidence rating of 2.3. RN indicated that he "mostly tried to guess" in this task.

\section{Partial calendar}

Single calendar pages, each displaying a particular year and month, were shown briefly to the participants. Following calendar page presentation, they were asked several other date questions from that same year. Before viewing the calendar pages, DG performed at chance $(13.9 \%$ correct, $10 / 72)$, with a mean confidence rating of 1.0. Following a 1-sec exposure to the calendar pages, his performance improved to $94.4 \%$ (68/72) with a mean confidence rating of 5.0.

$\mathrm{RN}$ had more difficulty with this task. Before viewing the calendar pages, RN performed at chance $(20.0 \%$ correct, $4 / 20)$ with a mean confidence rating of 2.1. Following 10- to 30-sec exposures to the calendar pages, he now scored only seven of 20 correct $(35.0 \%)$ with a mean confidence rating of 2.6. Although he performed poorly, his score was above chance $(P<0.04$; binomial test).

\section{Learning tasks}

We assessed the participants' ability to recall lists of items belonging to particular categories (either dates, times of day, threeword sentences, or digits). DG performed better than controls on the date learning and times-of-day learning tasks, but poorer on the sentence and number learning tasks (Fig. 4A). For date learning, his mean score across the five trials was above the $95 \%$ confidence interval calculated from the control scores. For sentence learning, his score was below the $95 \%$ confidence interval calculated from control scores. RN performed better than controls on all four tasks, scoring outside the $95 \%$ confidence interval on all but the sentence learning task (Fig. 4B).

\section{Memory for events}

DG was asked to recall past personal events occurring 1 to $7 \mathrm{yr}$ prior to the date of testing. DG accurately recalled the events documented on the receipts for 15 of 25 dates (mean confidence rating $=5.0$ ). For six of these 15 dates, he also provided additional information beyond what could be confirmed by the receipt. For instance, for one date, he correctly described the event

\section{A}

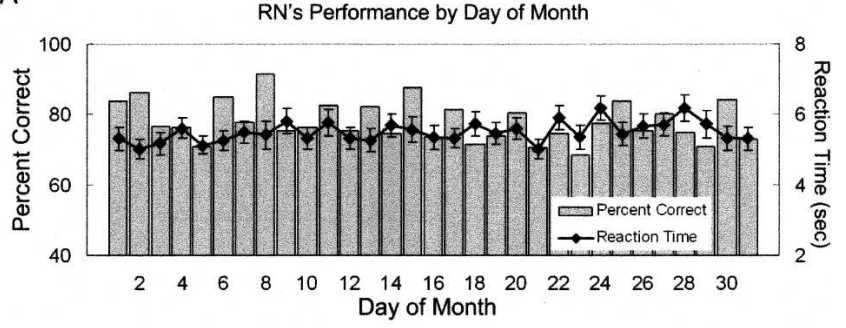

B

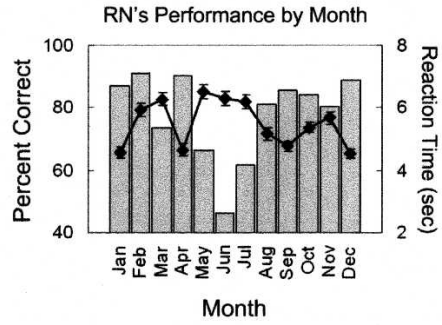

C

Figure 3. RN's performance (reaction time and percent correct) by day of the month $(A)$, month of the year $(B)$, and day of the week (C). Brackets show standard error of the mean. 
A
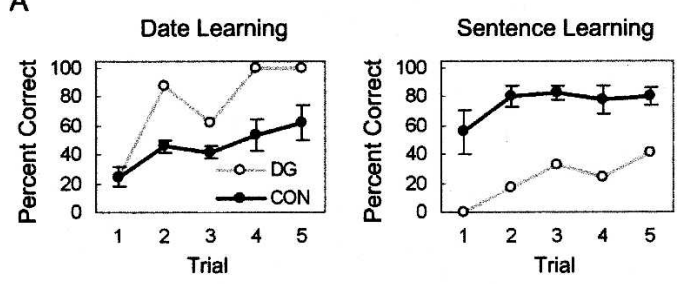

Time Learning
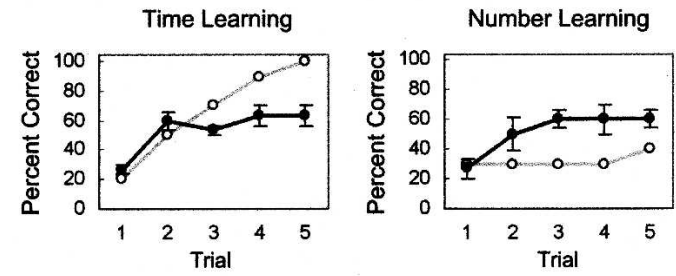

B

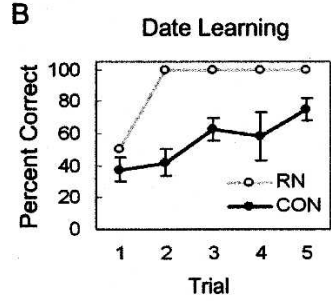

Time Learning
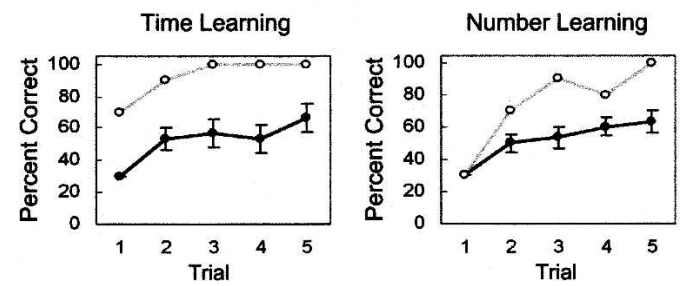

Figure 4. Performance on four learning tasks. ( $A$ ) DG performed better than controls $(n=3)$ on date and time learning, but worse on sentence and number learning. (B) RN performed better than controls $(n=3)$ in all four tasks. Brackets show standard error of the mean.

on the receipt and also correctly pointed out that he visited our laboratory that same day. For six questions involving the 10 dates that DG did not confirm, he claimed that he was not present at the event documented on the receipt (e.g., he said that he did not go out to dinner that night; mean confidence rating $=3.7$ ). His father stated that DG might be correct in these cases, and we could not obtain additional information. Finally, for four of the dates that DG did not confirm, he stated that the date on the receipt was wrong, and he described other events for those days that could not be confirmed or denied (mean confidence rating $=4.5$ ). We also asked DG to recall the dates of the previous testing sessions, and he accurately recalled the dates of the most recent seven sessions (from 1998 to 2003) before losing interest in the task.

\section{Discussion}

We acquired large data sets of calendar performance from two autistic calendar savants, DG and RN, and analyzed their errors and reaction times. We also administered two additional tests of calendar ability and a learning test. DG and RN were able to respond accurately to calendar dates from only a limited range of years (DG: 1985-2007; RN: 1961-2007). DG's reaction time and accuracy were better for the first day and last day of the month compared to all other days. RN's performance was similar across the days of the month but was poor overall for the months of May, June, and July.

In the date pairs task, only DG was able to answer questions about the calendar of a particular year after being given two dates and corresponding days of the week from that year. DG also performed well when he was asked to answer questions about the calendar following a 1-sec exposure to a single month from a calendar page (partial calendar task). In contrast, RN performed only modestly above chance levels even after 10- to 30-sec exposures to single calendar pages. Finally, DG was able to learn dates and times of day better than age-matched controls but did more poorly than controls at learning sentences and numbers (Fig. 4). DG also performed numerically better than controls at learning clock times but poorer at learning sentences and numbers. RN outperformed his controls on all four tests.

For both savants, the limited range of years for which calendar information was available corresponded roughly to years during their lifetimes. Their abilities extended only a few years before their births and several years into the future. It is possible that this limited range of ability reflects their access to these calendars. Importantly, RN's range of years encompassed all 14 calendar year templates. At the time of his final testing (at age 15), DG's knowledge encompassed 12 of the 14 possible calendar templates (and partially covered an additional calendar year template). On this occasion, DG's father told us that his son was no longer interested in studying calendars, and instead had begun to memorize U.S. highway maps and weather in various cities.

Although both RN and DG had complete or nearly complete knowledge of the 14 possible calendar templates, they did not use yearly regularities of the calendar to extend their abilities beyond the limited span of years that defined their knowledge. Some savants who possess knowledge of calendar years well into the past and future do so by recognizing that the calendar repeats every $28 \mathrm{yr}$ (i.e., the 28-year rule) (O'Connor and Hermelin 1984; Hermelin and O'Connor 1986; Ho et al. 1991; Thioux et al. 2006). These individuals apply the necessary additions or subtractions to arrive at a year that falls within the span of their abilities. It seems likely that neither DG nor RN recognized this calendar regularity. Indeed, the range of years that defined DG's calendar expertise did not extend as far as 28 yr. Similarly, although RN was able to answer calendar questions from several years in the 1930s, 1940s, and 1950s, he answered questions incorrectly from other years within these same decades.

DG's better performance for both reaction time and accuracy on questions about the first and last day of the month is not compelling evidence for the use of "anchor" dates from which to calculate other dates (Rosen 1981). We expected that anchor dates would not only be remembered more accurately and more quickly, but that a pattern of monotonically increasing errors and reaction times would be observed with increasing distance from the anchors. Because this pattern was not observed, we suggest that DG simply had learned these particular dates better than other dates.

Testing carried out before and after DG acquired calendar knowledge for the year 1993 provides further evidence against an anchor date strategy. At a time when his performance for 1993 averaged $26.1 \%$ correct overall, he was $83.3 \%$ accurate for questions about the first day of the month and 50\% accurate for questions about the last day of the month. If these two days in each month were being used as anchor dates, he should have performed better on the other days of the month.

We suggest that DG's abilities are based on his memorizing the structure of the calendar, possibly relying on visual imagery. The spatial layout of the calendar makes the first and last of the month more salient and presumably easier to remember. When attempting to recall a specific date, DG may first retrieve the 
specific calendar month for that year from memory. By drawing on its more salient features (i.e., the first and last days of the month), he can perform better on these dates compared to dates in the middle of the month. As there is no evidence for orderly calculation from these dates (e.g., increased latencies with increasing distance), we suggest instead that DG may be scanning his visual image of the month in order to "read" the answer. Consistent with this proposal, DG's excellent performance on the partial calendar task suggests that he has a good visual memory of the calendar. He was able to determine which calendar template he was looking at on the basis of a brief 1-sec visual presentation of a single calendar page (i.e., one month). Similarly, DG's excellent performance on the date pairs task is consistent with his having a visual memory of calendar structure. However, we cannot exclude the possibility that he is also using verbal memory strategies.

We suggest that RN organized and represented his calendar information differently than DG did. RN had difficulty with the partial calendar task even after 30-sec exposures to a calendar page, and he failed the date pairs task altogether. While it is unclear how RN accomplished his calendar feats, it appears that he may have also been relying on memorization, but memorizing that was incomplete (e.g., poor performance for May, June, and July dates) and that did not achieve an integrated representation of calendar relationships. Alternatively, RN's difficulty could arise because he cannot determine dates for a month that is different from the month of a date that he is given.

We also emphasize that the use of anchor dates, and calculation from anchor dates, is difficult to rule out entirely, particularly if different anchor dates were used for each month and/or year. Although logically possible, this idea is unlikely in the case of DG. DG had fast reaction time and high accuracy for the first and last days of the month. These days would have been his most likely anchor dates, and we found no evidence that calculations were being made based on these dates (i.e., no gradients in accuracy or latency as he was asked about more distant dates). For $\mathrm{RN}$, we found no particular dates with increased accuracy and fast reaction times. It is therefore possible that he used different anchor dates for each month and/or year, although this strategy seems, intuitively, to be more burdensome than memorizing the calendar pattern.

It is also interesting that DG had highly accurate memory for past personal events, an ability that has rarely been documented (but see Parker et al. 2006). To the extent that past events could be verified by old receipts and by records of previous laboratory visits, DG was remarkably accurate at recalling seemingly common-place personal events from his past. It is clear that DG has a propensity toward memorization.

What factors might influence the early development of calendar savant abilities? The calendar has an inflexible, predictable, and highly repetitive structure, characteristics to which the autistic individual is drawn. These same rigid and repetitive features are often seen in other domains where autistic individuals focus their interest-for example, schedules, maps, computers, and facts. It is also true that an interest in calendar information is supported by the cognitive style and by the cognitive strengths of individuals with autism, which include detail-oriented processing, sustained attention, an inclination for rote learning, and interest in rule-based systems (Allen and Courchesne 2001; Baron-Cohen et al. 2003; Happe and Frith 2006).

In summary, the findings were different for DG and RN. Although the two savants were similar in that their calendar knowledge was limited to only a few decades, they differed markedly in their patterns of calendar performance (DG was better at answering questions about the beginnings and ends of months; $\mathrm{RN}$ was better at the beginnings and ends of years). Furthermore, they differed in the extent to which they could answer particular questions about the calendar (DG was good at the partial calendar task and at the date pairs task; RN was not). Both individuals appeared to work from memorization, not from calculation, although memory seems less thorough for RN. These findings emphasize that the calendar abilities exhibited by calendar savants do not depend on one particular method, and that there are multiple strategies that can underlie this remarkable ability.

\section{Methods}

\section{Participants}

DG is a right-handed male who met the criteria for a diagnosis of autism based on the Autism Diagnostic Observation Schedule (ADOS) (Lord et al. 2000) and the Autism Diagnostic InterviewRevised (ADI-R) (Lord et al. 1994), administered by an experienced clinical psychologist. He was first tested in 1997 when he was $9 \mathrm{yr}$ old and on two other occasions when he was 13 and 15 yr old. He obtained a Verbal IQ score of 58 and a Performance IQ score of 53 (Full Scale IQ = 52) on the Wechsler Intelligence Scale for Children-III (WISC-III). On the Wide Range Achievement Test 3 (WRAT3), he performed in the 18th, 7th, and 9th percentile in reading, spelling, and arithmetic, respectively.

$\mathrm{RN}$ is a left-handed male who also met the criteria for a diagnosis of autism based on administration of the ADOS and ADI-R by an experienced clinical psychologist. He was first tested in 1997 when he was 33 yr old. On the Wechsler Adult Intelligence Scale-Revised (WAIS-R), he obtained a Verbal IQ of 98, a Performance IQ of 114 (Full Scale IQ = 104). On the Wide Range Achievement Test Revised 2 (WRAT-R2), he performed in the 45th, 50th, and 90th percentile in reading, spelling, and arithmetic, respectively.

Six healthy male controls (three matched for age with DG and three matched for age with $\mathrm{RN}$ ) were given the four learning tasks described below.

\section{Calendar dates}

Testing was done in the laboratory and at the participants' houses. Dates in the form of "Month, Day, Year" appeared on a computer screen (e.g., September 20, 1996), and the participants were asked to respond with the day of the week that corresponded to the given date (i.e., Friday). Reaction time was measured by computer from the appearance of the date on the screen to the onset of a vocal response. Testing proceeded in blocks of 25 dates (1-9 blocks/session). Each block consisted of randomly selected dates covering several years. Initial testing focused on the time periods for which the participants appeared to have the best knowledge. In total, DG participated in 10 sessions during $1.5 \mathrm{yr}$, and data were obtained for 1399 dates, covering the period 1993-1998 (tested in 1997-1998). Four years and again 6 yr after initial testing, at the age of 13-15, DG was tested on an extended list of calendar years, from 1979-2018 (tested in late 2001 and early 2003). At these times, he participated in a total of four sessions, and data were obtained for 492 additional dates.

RN completed 20 sessions during 1.5 yr. Data were obtained for 1730 dates from 1971-1999, and (with fewer observations per year) for another 780 dates from 1900-1970 and 2000-2020 (all tested in late 1997-early 1999).

\section{Date pairs}

In one to two sessions during 2003, two dates from the same year and their corresponding days of the week were read aloud to the participants (e.g., "January 22 was a Wednesday, and October 5 was a Sunday"). One of the dates was always from January or February, and the other was always from March or later. This procedure ensured that there was always enough information to determine if the year was a leap year or not. After the date pairs were read, participants were asked to provide the days of the week for four or five other dates from that same year (e.g., "What day of the week is June 6?") and to provide confidence ratings (1-5) for their answers. DG was tested with 16 date pairs (result- 
ing in 79 questions), and RN was tested with 12 date pairs (60 questions).

\section{Partial calendar}

In 2003, single calendar pages, each displaying a particular year and month, were shown briefly to the participants (e.g., all $31 \mathrm{~d}$ of May 2063). Before the test, we confirmed that the years being tested were outside the range of their abilities. DG was shown each calendar page for $1 \mathrm{sec}$, and RN was shown each page for 10 to $30 \mathrm{sec}$. (In preliminary testing, RN had difficulty with this task. In the end, we tested him after longer presentation times, because after 10 to $30 \mathrm{sec}$ he would usually indicate that he was ready to answer questions.) After viewing each calendar page, participants were given four to five different dates (i.e., a date from a different month), and they were asked to provide the corresponding day of the week and to give confidence ratings (1-5) for their answers. DG was tested on 72 different dates, and $\mathrm{RN}$ was tested on 20 different dates.

\section{Learning tasks}

Four different tests were constructed, each consisting of four to six items (dates, times of day, three-word sentences, or digits). The items in one of the tests (e.g., dates) were first read aloud to the participants. There were four dates such as "August 11, 1984"; five times of day, such as "10:02 p.m."; six three-word sentences, such as "bird buried treat"; and five three-digit numbers, such as "847." Immediately following presentation of the items, participants were asked to recall them. This same procedure was repeated for a total of five learning trials. The items were given in a different order on each presentation. The four different types of tests were also given in a mixed order to the different participants. Two versions of each test were given, and each participant's score was his average score on the two versions.

\section{Memory for events}

DG's father reported that DG could remember events from specific dates since the age of 8 . To explore this, his father provided 25 receipts that documented specific events (e.g., dinners) from the years 1995-2002 (tested in 2003). DG was then given the date (e.g., March 23, 1998) and asked to recall the events of that date. If his answer did not relate to the information on the receipt, we prompted him with follow-up questions (e.g., where did you go for dinner that night?).

\section{Acknowledgments}

We thank DG, RN, and their families for graciously donating their time for this research. This study was supported by the Medical Research Service of the Department of Veterans Affairs, the National Institute of Mental Health, and the Metropolitan Life Foundation. We thank Daniel Schacter, August Tuan, Jennifer Frascino, Joyce Zouzounis, and Cindy Carter for advice and assistance.

\section{References}

Allen, G. and Courchesne, E. 2001. Attention function and dysfunction in autism. Front. Biosci. 6: D105-D119.

Baron-Cohen, S., Richler, J., Bisarya, D., Gurunathan, N., and Wheelwright, S. 2003. The systemizing quotient: An investigation of adults with Asperger syndrome or high-functioning autism, and normal sex differences. Philos. Trans. R. Soc. Lond. B Biol. Sci. 358: 361-374.

Happe, F. and Frith, U. 2006. The weak coherence account: Detail-focused cognitive style in autism spectrum disorders. J. Autism Dev. Disord. 36: 5-25.

Hermelin, B. and O'Connor, N. 1986. Idiot savant calendrical calculators: Rules and regularities. Psychol. Med. 16: 885-893.

Hill, A.L. 1975. An investigation of calendar calculating by an idiot savant. Am. J. Psychiatry 132: 557-560.

Ho, E.D., Tsang, A.K., and Ho, D.Y. 1991. An investigation of the calendar calculation ability of a Chinese calendar savant. J. Autism Dev. Disord. 21: 315-327.

Iavarone, A., Patruno, M., Galeone, F., Chieffi, S., and Carlomagno, S. 2007. Brief report: Error pattern in an autistic savant calendar calculator. J. Autism Dev. Disord. 37: 775-779.

Lord, C., Rutter, M., and Le Couteur, A. 1994. Autism Diagnostic Interview-Revised: A revised version of a diagnostic interview for caregivers of individuals with possible pervasive developmental disorders. J. Autism Dev. Disord. 24: 659-685.

Lord, C., Risi, S., Lambrecht, L., Cook Jr., E.H., Leventhal, B.L., DiLavore, P.C., Pickles, A., and Rutter, M. 2000. The Autism Diagnostic Observation Schedule-Generic: A standard measure of social and communication deficits associated with the spectrum of autism. J. Autism Dev. Disord. 30: 205-223.

Mottron, L., Lemmens, K., Gagnon, L., and Seron, X. 2006. Non-algorithmic access to calendar information in a calendar calculator with autism. J. Autism Dev. Disord. 36: 239-247.

O'Connor, N. and Hermelin, B. 1984. Idiot savant calendrical calculators: Maths or memory? Psychol. Med. 14: 801-806.

Parker, E.S., Cahill, L., and McGaugh, J.L. 2006. A case of unusual autobiographical remembering. Neurocase 12: 35-49.

Rosen, A.M. 1981. Adult calendar calculators in a psychiatric OPD: A report of two cases and comparative analysis of abilities. J. Autism Dev. Disord. 11: 285-292.

Thioux, M., Stark, D.E., Klaiman, C., and Schultz, R.T. 2006. The day of the week when you were born in $700 \mathrm{~ms}$ : Calendar computation in an autistic savant. J. Exp. Psychol. Hum. Percept. Perform. 32: 1155-1168.

Young, R.L. and Nettelbeck, T. 1994. The "intelligence" of calendrical calculators. Am. J. Ment. Retard. 99: 186-200.

Received May 29, 2007; accepted in revised form June 30, 2007. 


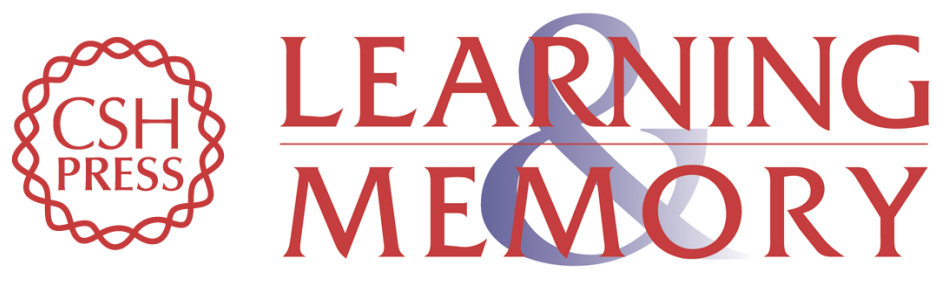

\section{An analysis of calendar performance in two autistic calendar savants}

Daniel P. Kennedy and Larry R. Squire

Learn. Mem. 2007, 14:

Access the most recent version at doi:10.1101/Im.653607

\begin{tabular}{cc}
\hline License & $\begin{array}{c}\text { Receive free email alerts when new articles cite this article - sign up in the box at the } \\
\text { top right corner of the article or click here. }\end{array}$ \\
\hline
\end{tabular}

